\title{
A DNA Coding Scheme for Searching Stable Solutions
}

\author{
Intaek Kim ${ }^{1}$, HeSong Lian ${ }^{1}$, and Hwan Il Kang ${ }^{2}$ \\ ${ }^{1}$ Department of Communication Eng., Myongji University, \\ 449-728, Yongin, South Korea \\ kit@mju.ac.kr, hslian@hotmail.net \\ ${ }^{2}$ Department of Information Eng., Myongji University, \\ 449-728, Yongin, South Korea \\ hwan@mju.ac.kr
}

\begin{abstract}
This paper presents a novel method for searching stable solutions using a DNA coding scheme. Often there is more than one solution that satisfies the system requirements. These solutions can be viewed as extremes in the multimodal function. All extremes are not the same in that some of them are sensitive to noise or perturbation. This paper addresses the method that selects a solution that meets the system requirements in terms of output performance and is tolerant to the perspective noise or perturbation. A new method called a gradient DNA Coding is proposed to achieve such objectives. A numerical example is presented and comparing DNA coding with genetic algorithm is also given.
\end{abstract}

Keywords: DNA coding, genetic algorithms, gradient DNA coding.

\section{Introduction}

The genetic algorithm (GA) has been widely used in many optimization problems. The GA offers an efficient way to search a global solution in the multimodal function [1][2]. The multimodal function may have several solutions, but some of them are very sensitive to small perturbations of their parameter values. They may be not good solutions in certain situations.

In many optimization tasks, there is a need to find solutions whose performance will not change much due to small variation of the parameter values. In this paper, we define a stable solution as one whose variation results in a small amount of change in output performance that satisfies the system requirement. We propose a new coding method for searching stable solutions. It is based on the biological DNA and a mechanism of artificial DNA [4][5]. A gradient information is utilized to find solutions and it is named a gradient DNA coding method. In addition, the comparison between methods using the simple DNA coding and the gradient DNA coding is presented. In the next section, the DNA coding method is described. The proposed algorithm is given in section 3 and it is followed by simulation to show the effectiveness of the proposed method. The final section is followed for the conclusion and future work. 


\section{DNA Coding Method}

The biological DNA consists of nucleotides which have four bases, Adenine(A), Guanine(G), Cytosine(C) and Thymine(T)[4][5][6]. A messenger RNA (mRNA) is first synthesized from the DNA. In the synthesis of RNA, each base is translated into the complementary base and the unused parts are cut out. This operation is a splicing. After this splicing the mRNA is completed. Three successive bases called codons are allocated sequentially in the mRNA. These codons are the codes for amino acids. 64 kinds of codons correspond to 20 kinds of amino acids as shown in Table 1 . The details of translation into amino acid from codons are omitted here. This allocation of amino acid makes proteins, and proteins make up cells.

A Figure 1 shows an example of the DNA chromosome and its translation mechanism. A gene begins with the start codon ATG, and closes with end codons TAG, TAA or TGA [6]. The Figure 1 indicates that Gene1 consists of eight condons: CGG, CGT, ..., TCC and they are translated into amino acids: Arg, Arg, ..., Ser, respectively. Each amino acid has a number from 0 to 9 as shown in Table 2. The sum of the acids value represents gene's value and it is plugged in as a parameter value. For example, Gene 2 consists of acids: Arg, Gly, Phe, Leu, Ala, Ser and Gly and its value becomes 26.25 by adding the value of each acids.

Table 1. RNA(DNA) Codon and amino acid

\begin{tabular}{|c|c|c|c|c|c|c|c|c|c|}
\hline & \multicolumn{2}{|c|}{$\mathbf{T}$} & \multicolumn{2}{|c|}{$\mathrm{C}$} & \multicolumn{2}{|c|}{$\mathbf{A}$} & \multicolumn{2}{|c|}{$\mathbf{G}$} & $\mathbf{T}$ \\
\hline \multirow[t]{4}{*}{$\mathbf{T}$} & TTT & \multirow[t]{2}{*}{ Phe } & TCT & \multirow{4}{*}{ Ser } & TAT & \multirow[t]{2}{*}{ Tyr } & TGT & \multirow[t]{2}{*}{ Cys } & \\
\hline & TTC & & TCC & & TAC & & TGC & & $\mathbf{C}$ \\
\hline & TTA & \multirow{6}{*}{ Leu } & TCA & & TAA & \multirow{2}{*}{ Stop } & TGA & Stop & $\mathbf{A}$ \\
\hline & TTG & & TCG & & TAG & & TGG & Trp & $\mathbf{G}$ \\
\hline \multirow{4}{*}{$\mathbf{C}$} & CTT & & CCT & \multirow{4}{*}{ Pro } & CAT & \multirow{2}{*}{ His } & CGT & \multirow{4}{*}{ Arg } & $\mathbf{T}$ \\
\hline & CTC & & $\mathrm{CCC}$ & & $\mathrm{CAC}$ & & CGC & & $\mathbf{C}$ \\
\hline & CTA & & $\mathrm{CCA}$ & & CAA & & CGA & & $\mathbf{A}$ \\
\hline & CTG & & CTG & & CAG & Gln & CGG & & G \\
\hline \multirow{4}{*}{$\mathbf{A}$} & ATT & \multirow{3}{*}{ Ile } & ACT & \multirow{4}{*}{ Thr } & AAT & \multirow{2}{*}{ Asn } & AGT & \multirow{2}{*}{ Ser } & $\mathbf{T}$ \\
\hline & ATC & & ACC & & AAC & & AGC & & $\mathrm{C}$ \\
\hline & ATA & & ACA & & AAA & I ve & AGA & Aro & $\mathbf{A}$ \\
\hline & ATG & Met & ACG & & $\mathrm{AAG}$ & Lys & $\mathrm{AGG}$ & Arg & $\mathbf{G}$ \\
\hline \multirow{4}{*}{ G } & GTT & \multirow{4}{*}{ Val } & GCT & \multirow{4}{*}{ Ala } & GAT & Asn & GGT & \multirow{4}{*}{ Gly } & $\mathbf{T}$ \\
\hline & GTC & & GCC & & GAC & Asp & GTC & & $\mathbf{C}$ \\
\hline & GTA & & GCA & & GAA & & GGA & & $\mathbf{A}$ \\
\hline & GTG & & GCG & & GAG & Glu & GGG & & $\mathbf{G}$ \\
\hline
\end{tabular}


Table 2. The value for each amino acid

\begin{tabular}{|c|c|c|c|c|c|c|r|}
\hline Phe & 0.25 & Pro & 0.50 & His & 0.75 & Glu & 1.00 \\
\hline Leu & 1.25 & Thr & 1.50 & Gln & 1.75 & Cys & 2.00 \\
\hline Ile & 3.25 & Ala & 2.50 & Asn & 2.75 & Trp & 3.00 \\
\hline Met & 3.25 & Tyr & 3.50 & Lys & 3.75 & Arg & 4.00 \\
\hline Ser & 4.25 & Val & 4.50 & Asp & 4.75 & Gly & 5.00 \\
\hline
\end{tabular}

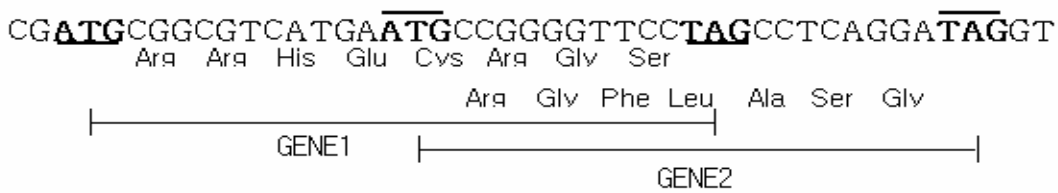

Fig. 1. The example of genes overlapping and chromosome translation mechanism

\section{Algorithm}

In this paper, the proposed algorithm can be summarized in 7 steps as follows:

\section{Step 1: Initialization}

Determine the population size, the chromosome length, and probabilities of crossover and mutation. The simple GA (genetic algorithm) is initialized with the binary number $(0,1)$, but the DNA coding is initialized with four bases: $\mathrm{A}$ (adenine), $\mathrm{T}$ (thymine), $\mathrm{G}$ (guanine) and C(cytosine).

\section{Step 2: Addition of the noise}

The noise is generated and added to parameters in the system. If a given population size is $n$, the parameter can be expressed as $X=\left(x_{1}, x_{2}, \cdots x_{n}\right)$ and the noise is given by $\Delta=\left(\delta_{1}, \delta_{2}, \cdots \delta_{n}\right)$ where $\delta_{k}$ has a random variable from the interval [0, 0.02]. Either $X+\Delta$ or $X-\Delta$ is considered as a noise-added parameter. This noise vector will be used in next step.

Step 3: Calculation of the average value of gradients

In this procedure, the gradients $S\left(x_{i}, x_{i}+\delta_{i}\right)$ and $S\left(x_{i}, x_{i}-\delta_{i}\right)$ will be obtained, so is the average value of absolute gradient, that is, $\left(\left|S\left(x_{i}, x_{i}+\delta_{i}\right)\right|+\left|S\left(x_{i}, x_{i}-\delta_{i}\right)\right|\right) / 2$. This value will be used in calculating the fitness function.

Step 4: Calculation of the fitness

The above average value will be taken into account in this step. A Gene with a larger gradient value has a smaller fitness value so that it can be degenerated in the next 
generation. Fitness values also include the object function's value as well as one obtained from the gradient information. The optimal fitness function requires two conditions: the high object function value and the low average absolute gradient value.

\section{Step 5: Selection}

The Roulette Wheel selection method is used. It is better than Tournament selection method to avoid the object function converging into local optimums.

Step 6: Crossover and mutation

The Elitist strategy is adopted as a crossover operator. It saves the best gene in every chromosome so that the best solution will never degenerate. The mutation is performed randomly by the given mutation probability.

Step 7: go to step 2 until the required conditions are satisfied.

\section{Simulation}

In order to demonstrate the capability of the proposed algorithm, we applied the algorithm to a 1-D function that is the multimodal function. In simulation, the parameters were kept constant with the mutation probability $p_{m}=0.02$, the crossover probability $p_{c}=0.6$, the population size $\mathrm{N}=60$, the maximum number of generation 50 . we performed 50 simulations for each experiment with randomly initializing the population.

1) Function $y_{1}$ : Consider a function $y_{1}$ [Fig. 2(a)], which has five unequal peaks in $\mathrm{t}$ he range $0 \leq x \leq 1$ and is a variant of the function used in [3]. It is defined as

$$
Y_{1}(x)=\left\{\begin{array}{lc}
e^{-2 \ln 2\left(\frac{x-0.1}{0.8}\right)^{2}}|\sin (5 \pi x)|^{0.5} & 0.4<x \leq 0.6 \\
e^{-2 \ln 2\left(\frac{x-0.1}{0.8}\right)^{2}} \sin ^{6}(5 \pi x) & \text { otherwise }
\end{array}\right.
$$

As shown in Fig. 2(a) the global optimum is located at $x=0.1$ with the function value 1.0. There are four sharp peaks. The third peak is broad compared to others and is located at $x=0.486$ with function value 0.715 .

Fig.2 (b), (c) shows a typical distribution of the individuals in the after 50 generations for the simple DNA coding method and the gradient DNA coding method. Fig.2 (d) shows a convergence process of the mean value of parameter $x$ in the population with trials. The simple DNA coding method converged at $x=0.1$, the center of the highest peak. The gradient DNA coding method converged to the stable peak ( $x=0.486$ ) zone. Indeed, we can observe from Fig.2 (d) that it approached the broad peak. 


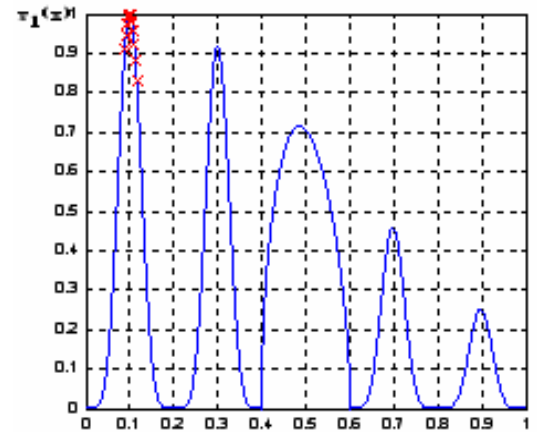

(a)

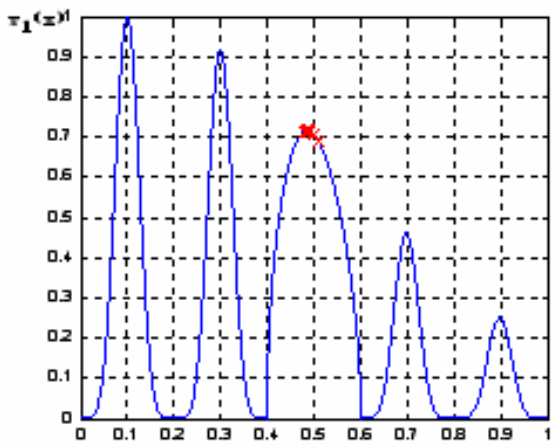

(c)

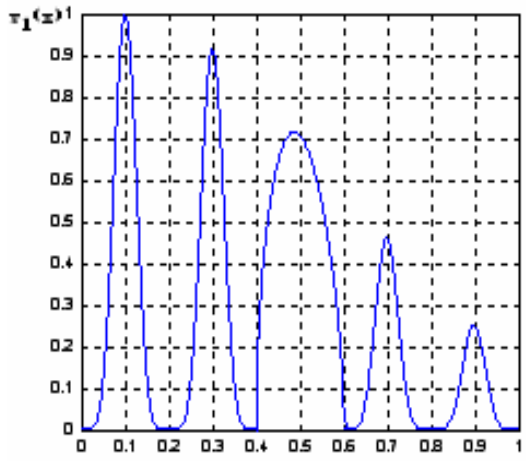

(b)

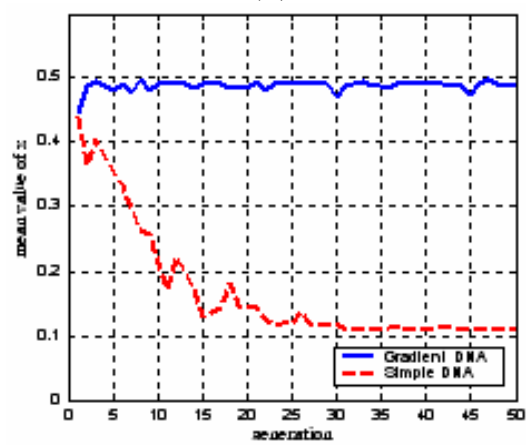

(d)

Fig. 2. (a) The original function $y_{1}$ (b) A typical distribution of the individuals in function $y_{1}$ after 50 generation for the simple DNA coding method (c) A typical distribution of the individuals in function $y_{1}$ after 50 generation for the gradient DNA coding method (d) The variation of mean (over the population) value of $x$ with function evaluations

\section{Conclusions}

This paper proposed a gradient DNA coding method, which extends the application of GA's to domains that require detection of stable solutions. The gradient DNA coding method was found that this approach can be effective when we want to detect more than one stable solutions on different peaks.

The future work will focus on analyzing the behavior of gradient DNA coding method on more complicated problems where many peaks interact, evaluating the gradient DNA coding method on real-world problems.

\section{Acknowledgment}

This work was supported by grant No. (R01-1999-000-00226-0) from the Basic Research Program of the Korea Science \& Engineering Foundation. 


\section{References}

1. Zhijiang Guo, Hongtao Zheng, Jinping Jiang,: A powerful modified genetic algorithm for multimodal function optimization, , Proceedings of the American Control Conference, Vol. 4 , (2002) $3168-3173$

2. Park Chang-Su, Lee Hungu, Bang Hyo-Choong, Tahk Min-Jea: Modified Mendel operation for multimodal function optimization, Evolutionary Computation, Proceedings of the 2001 Congress on , Vol. 2 , (2001) $1388-1392$

3. Nasraoui, O.; Krishnapuram, R.: A novel approach to unsupervised robust clustering using genetic niching, Fuzzy Systems, 2000. FUZZ IEEE The Ninth IEEE International Conference on , Vol. 1 (2000) $170-175$

4. Wasiewicz, Piotr, Janczak, Tomasz, JMulaka, J.: the Inference via DNA Computing, Evolutionary Computation, 1999. CEC 99. Proceedings of the 1999 Congress on , Vol. 2,(1999) 988-993

5. Deaton, R., and et. Al: A DNA Based Implementation of an Evolutionary Search for Good Encodings for DNA Computation, Proc. IEEE Int. Conf. Evolution computation, Indianapolis, IN, USA, April, (1997) 267-271

6. Yoshikawa, Tomohiro, Furuhashi, Takeshi, Uchikawa Yoshiki: DNA Coding Method and a Mechanism of Development for Acquisition of Fuzzy Control Rules, Fuzzy Systems, 1996., Proceedings of the Fifth IEEE International Conference on , Vol. 3 , (1996) 2194 2200 